\title{
The Development of Mapping, Covering and Control Strategies for a Vacuum Cleaner Robot
}

\author{
Thi Thoa Mac*, Nguyen Thanh Hung \\ Hanoi University of Science and Technology, Hanoi, Vietnam \\ *Email: thoa.macthi@hust.edu.vn
}

\begin{abstract}
Modern households are becoming more and more convenient and intelligent by applying new technology to reduce the time spent on house chores. In this study, the authors proposed the mapping, covering strategies, and control algorithms for vacuum cleaner robot. The robot will automatically implement the cleaning task in a single pass. The sensor system includes infrared sensor, 9 Dof MPU 9250, Delta Lidar 2A, ultrasonic sensor to help robots navigate, build maps and detect obstacles. ROS system (Robot Operating System) is used to control and simulate vacuuming operation in real-world environments. The experiments are conducted in order to illustrate the superiority of the proposed approach.
\end{abstract}

Keywords: covering strategy, localization, mapping, cleaner robot, PID controller, system identification.

\section{Introduction}

In recent years, mobile robots have been developed in many active research areas such as precision agriculture, swarm robot [1], autonomous navigation [2], cleaning, tidying up, or doing the laundry in the domestic environment [3]. Cleaning tasks, in particular, are the most frequent household chores in modern environments. In household life, with the development of intelligent technology, robotic vacuum cleaners and other smart appliances are more and more popular [4]. A vacuum cleaner robot is an intelligent machine commonly used for cleaning floors and carpets by suction [5-6].

In order to save time for people, the cleaning strategies of the robot are investigated in various ways. Floor cleaning robots are expected to archive a market value of 2.5 billion by 2020 [7-8]. The remarkable market players consist of iRobot Roomba, Xiaomi, robot iLife. Normally, the cleaning robots are operated with random movement. However, there is no guarantee that the floor is fully covered [9]. Zheng et al. in [10] investigates Mocap system for the multiple floor cleaning robot platforms. A development of a low-cost Arduino cleaning robot using mapping algorithm is proposed in [11]. However, the proposed approach is not efficient because they neglect the unknown environment.

This work focuses on system identification, covering strategies, the controller and mapping for a low-cost vacuum cleaner robot. The proposed approach does not require information of the room map in advance, therefore it works in a real time effectively.

ISSN: 2734-9373

https://doi.org/10.51316/jst.150.ssad.2021.31.1.8

Received: 11 January 2021; accepted: 21 February 2021
This paper is organized as follows. In section 2 , we explain the design of the robot. In addition, the system identification, control, covering strategies and mapping are introduced. In section 3 , we give a brief description of experiment results and discussions. Finally, conclusion is provided in section 4 .

\section{System Identification, Control, Covering strategies and Mapping}

A description of the vacuum cleaner robot's main characteristics, robot model, system identification and controller design, sensory equipment, system setup and localization are presented in this section.

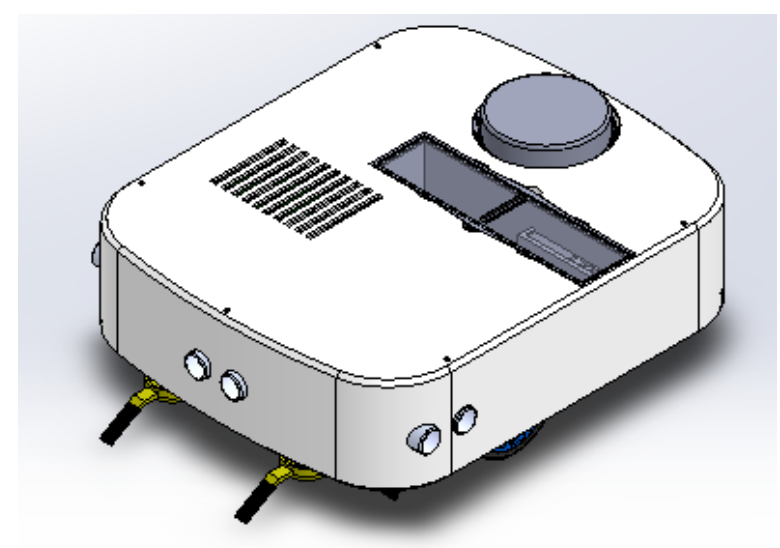

Fig. 1. The vacuum cleaner robot design.

\subsection{System Design}

The designed robot has a differential drive system with two independent wheels and a caster for stability as shown in Fig.1. The localization of the vacuum cleaner robot in the global coordinate $O x y$ is determined by the position of the mass center of 
robot $P(x, y)$; and the angle between the local frame $P x_{m} y_{m}$ and the global frame $\theta$ as depicted in Fig. 2.

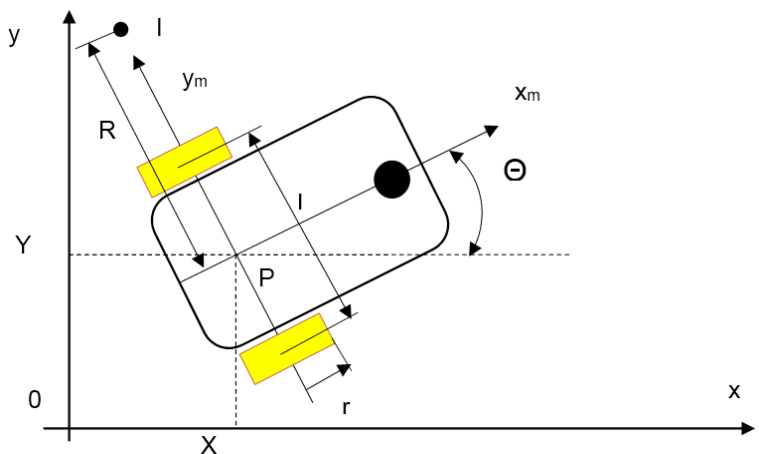

Fig. 2. The vacuum cleaner robot's kinematic model.

The robot's parameters include:

- $v_{t}$ : the linear velocity of the left wheel

- $\quad v_{p}:$ the linear velocity of the right wheel

- $\quad v$ : the linear velocity of the robot

- $\omega_{t}$ : the angular velocity of the left wheel

- $\quad \omega_{p}:$ the angular velocity of the right wheel

- $L$ : the distance between two wheels

- $R$ : the distance from the center of robot to the center of instantaneous velocity

- $\quad I$ : the center of the instantaneous velocity

The kinematic model of the robot is:

$$
\left[\begin{array}{c}
v_{x}(t) \\
v_{y}(t) \\
\dot{\theta}
\end{array}\right]=\left[\begin{array}{cc}
\frac{\cos \theta}{2} & \frac{\cos \theta}{2} \\
\frac{\sin \theta}{2} & \frac{\sin \theta}{2} \\
\frac{1}{L} & -\frac{1}{L}
\end{array}\right]\left[\begin{array}{c}
v_{P} \\
v_{t}
\end{array}\right]
$$

The robot is equipped with infrared/ultrasonic and lidar sensor. MPU sensor that determines the most accurate direction is used to guide the robot. Combined with lidar, infrared sensor is used to detect obstacles and evaluate different places. These sensors will send signals to the Raspberry Pi 3+ microcomputer for proper processing and navigation methods. During the operation, the Arduino microcontroller plays an intermediate role in the communication between the microcomputer with sensors and motors as shown in Fig. 3.

\subsection{System Identification}

In order to obtain the transfer function of the system, the characteristic of the open-loop system is investigated. A plot of the step response is shown in Fig. 4.

The time constant is chosen us:

$$
T=0.25(s)
$$

The transfer functions is:

$$
C(s)=\frac{130}{0.25 s+1}
$$

The PID controller parameters are determined by the root locus method as shown in Fig. 5 with $K_{p}=0.25$, $K_{i}=0.5$.

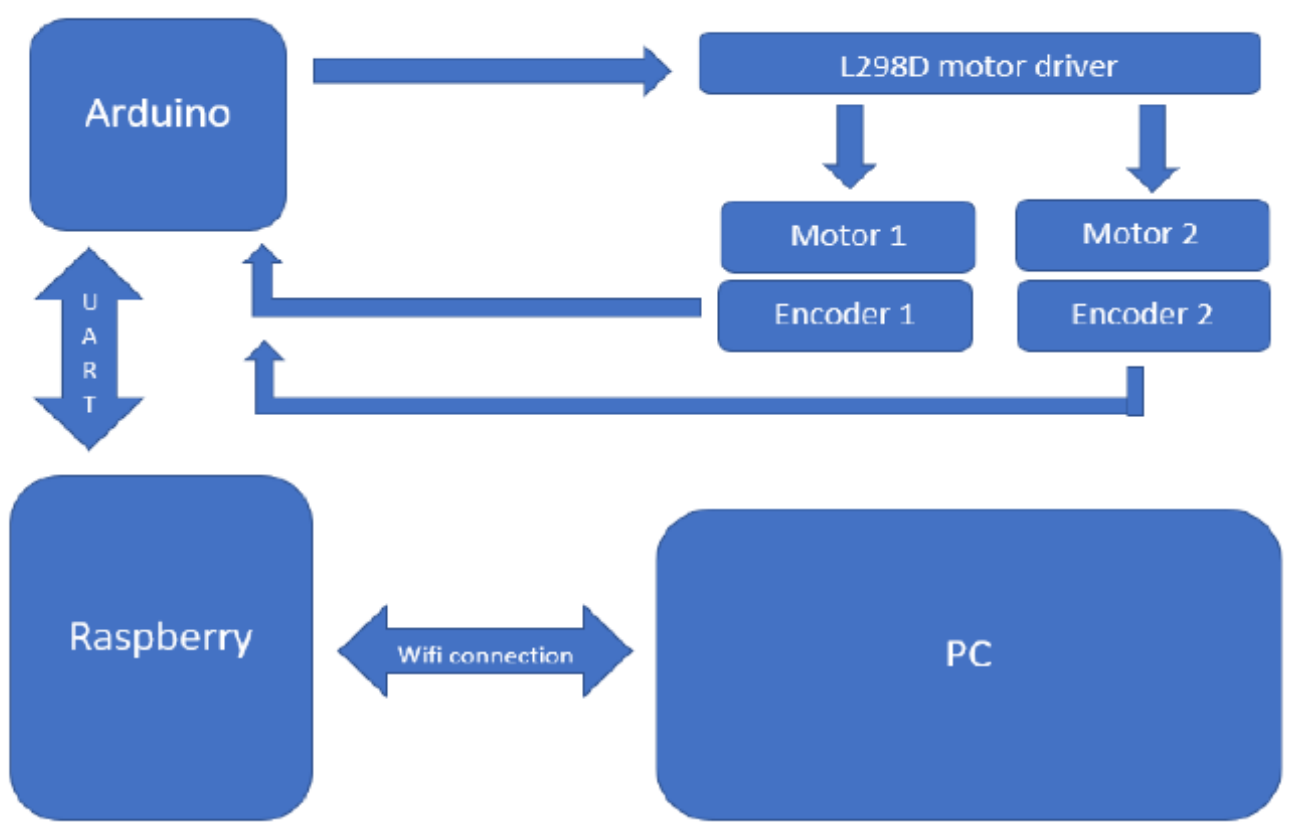

Fig. 3. The robot control circuit diagram. 
JST: Smart Systems and Devices

Volume 31, Issue 1, May 2021, 059-067

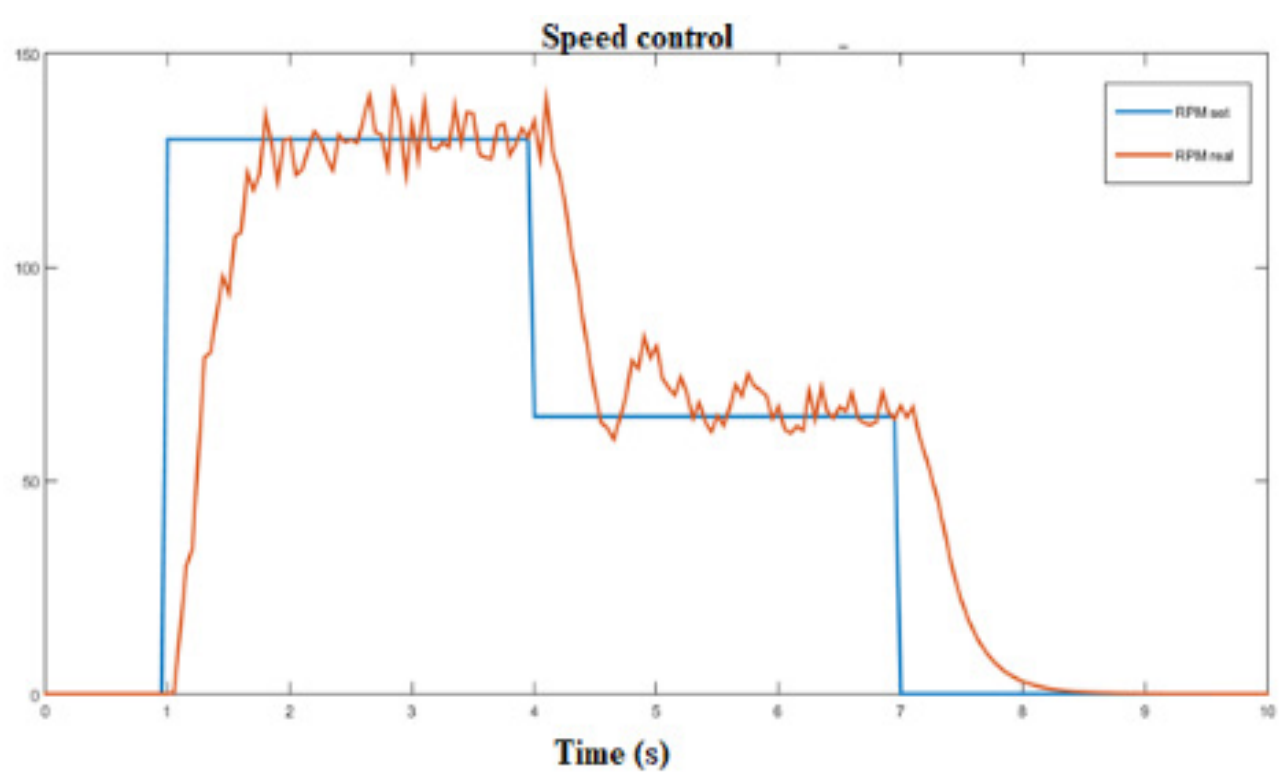

Fig. 4. The step response.

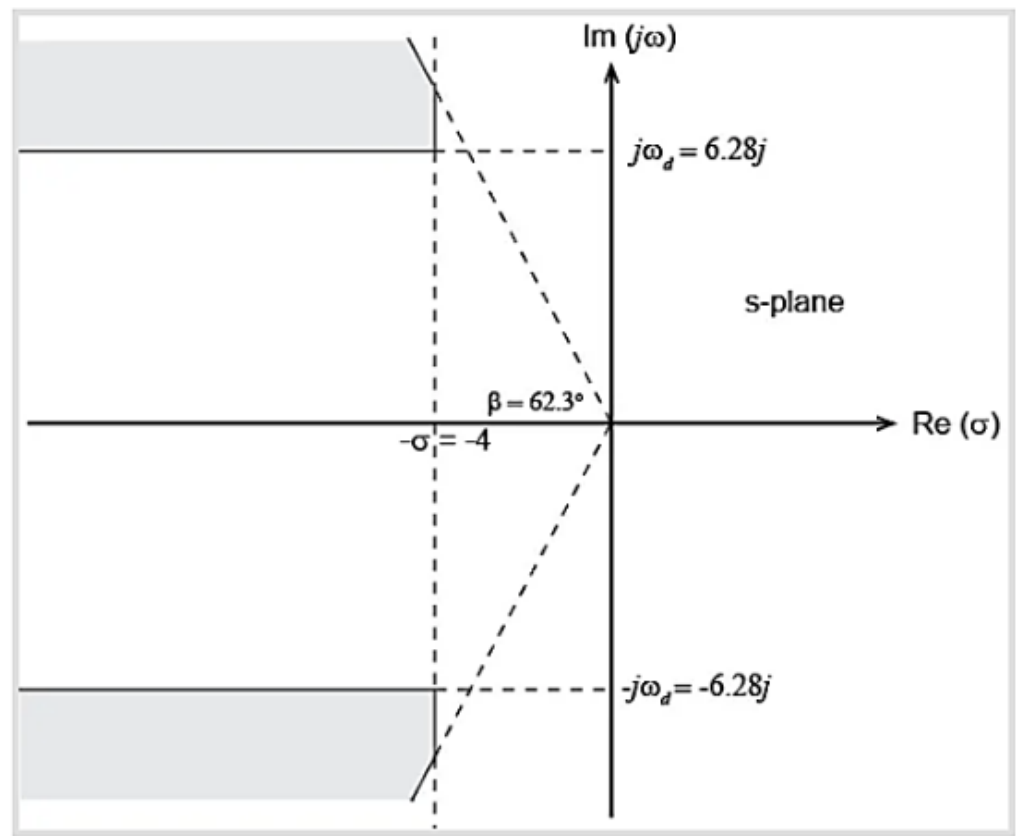

Fig. 5. The root locus of the system.

\subsection{Mapping}

Exploration for mapping in an unknown environment is an important task of autonomous navigation in general and for vacuum cleaner robot in particular. In this study, the method of detecting the border between the defined space and the undiscovered space is implemented to construct the map. The algorithm diagram is shown in Fig. 6.

When the robot moves to a border, it determines the features to create a new map by repeating the above process. The defined space is continuously expanded until it reaches the terminated condition. At this point, the map of the working environment is completely constructed.

\subsection{Localization}

In order to develop a reasonable path planning, the robot needs to locate its current position and direction in the working space as described in Fig. 7. In this study, the absolute positioning method based on Monte Carlo algorithm (Monte Carlo Localization) MCL is used to find the robot position.

The algorithm uses a set of $N$ random samples to determine the exact position of the robot, which is a discrete approximation of the robot's position.

$$
N=[[x, y, \theta], p]
$$


JST: Smart Systems and Devices

Volume 31, Issue 1, May 2021, 059-067

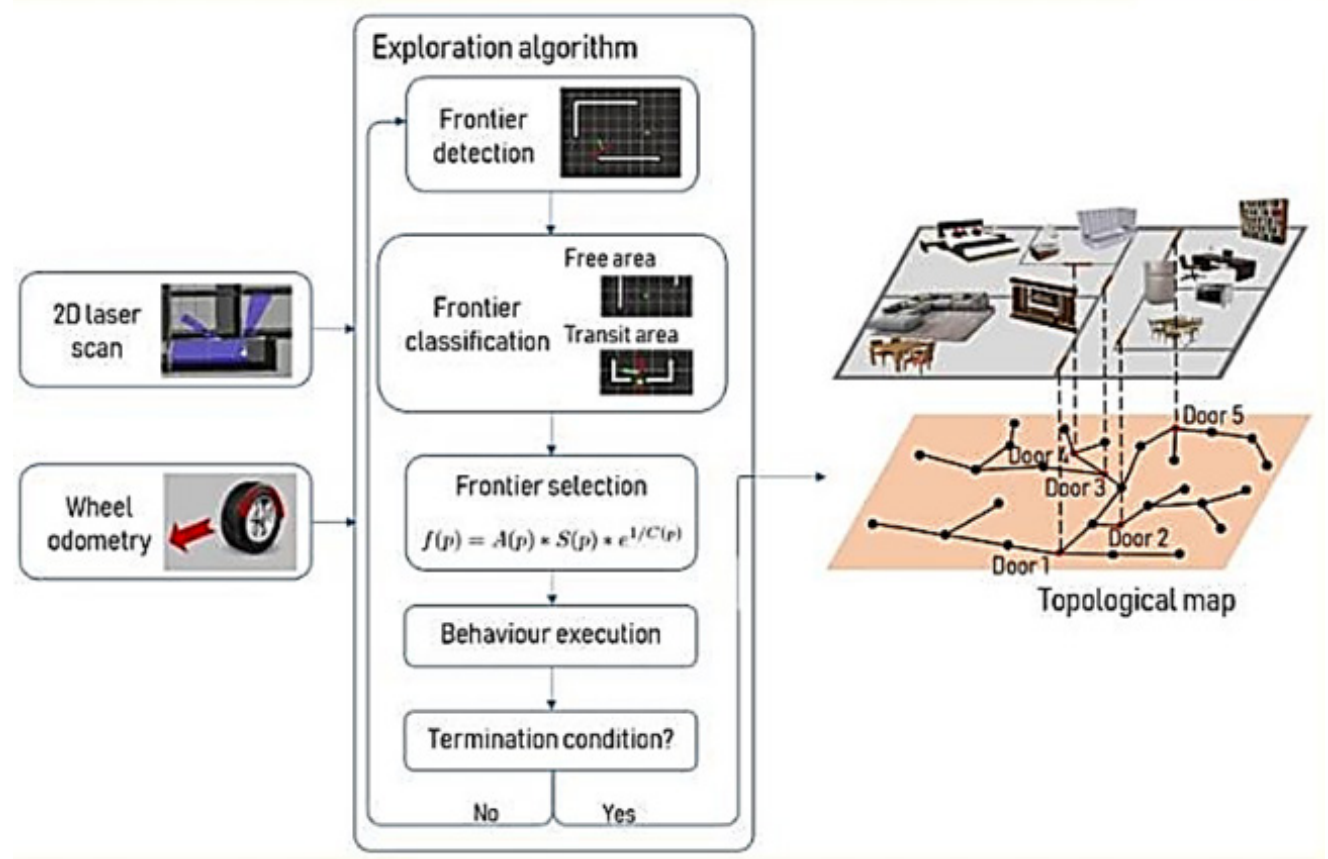

Fig. 6. Exploration diagrams and map construction.

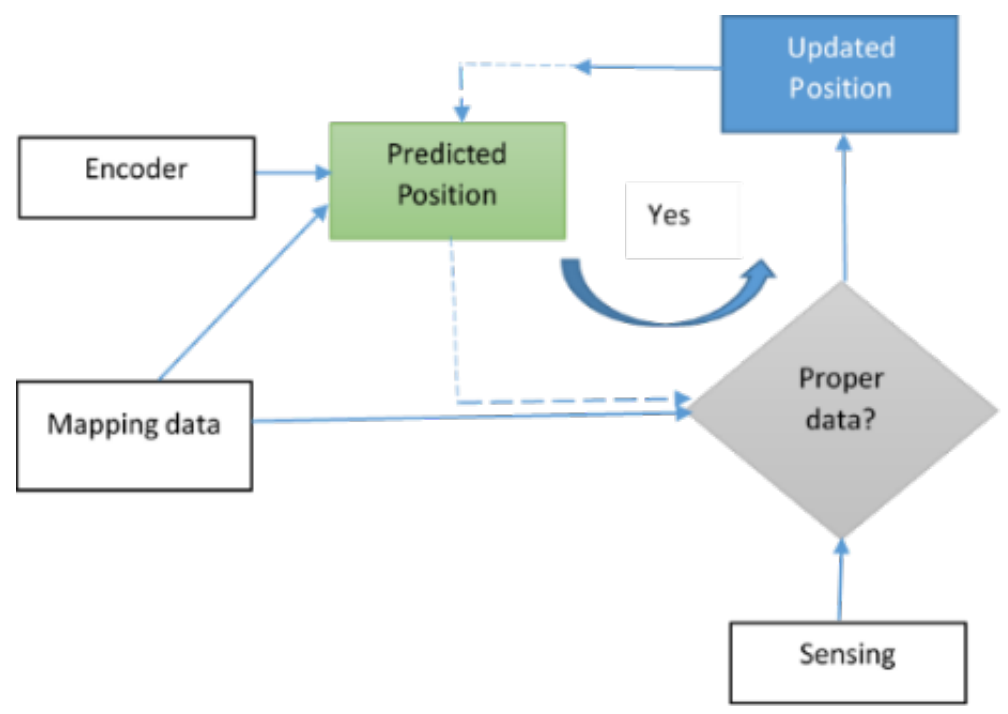

Fig. 7. Localization process.

where $[x, y, \theta]$ is the position and orientation in the working space, $p \geq 0$ is the discrete probability, with $\sum_{n=1}^{N} p_{n}=1$. For more detail, please refer [12].

\subsection{Covering Strategies}

In this subsection, we will discuss three different methods of covering a floor as shown in Fig. 8, 9, 10 . Having their advantages and disadvantages, the proposed solutions are particularly proper for different situations.

The robot moves from the starting position following an outward spiral as shown in Fig. 8a. When detecting an obstacle, the robot avoids and follows the spiral trajectory as shown in Fig. 8b. The robot can cover the required area with this trajectory. The time required to cover the entire area is reduced.

The zig-zag covering strategy is a paradigm shift proposition. The robot starts from a corner of the map and travels zig-zag until the entire map is covered. If an obstacle is in the robot's trajectory, it will move around the object as shown in Fig. 9.

The third solution is an inward spiral. With the starting position from the corner of the floor, the robot moves toward the center as shown in Fig. 10. Unlike the zig-zag model, this method can be derived from any starting point. Similar to the zig-zag method, when detecting an obstacle, the robot switches the obstacle ring and continues to follow the planned trajectory. 


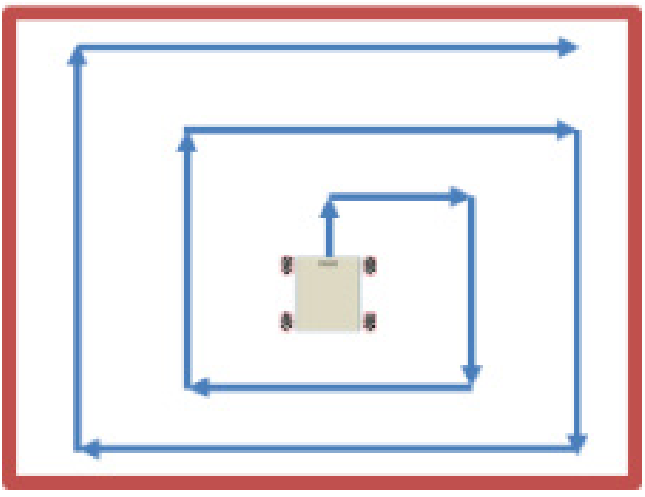

(a)

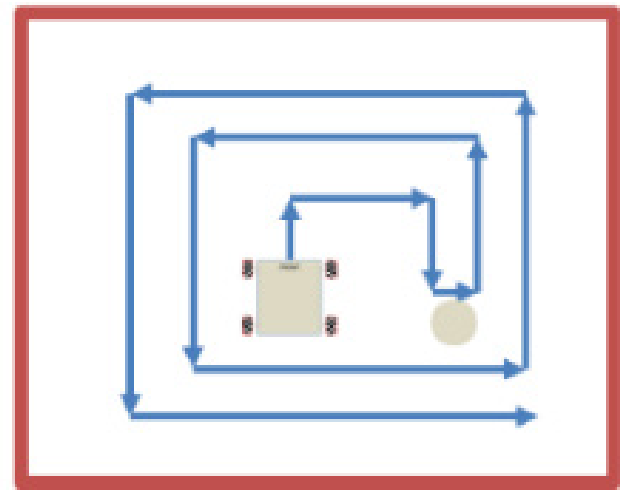

(b)

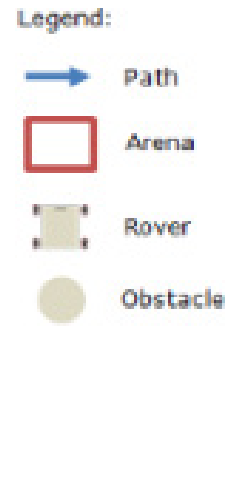

Fig. 8. The outward spiral covering strategies.

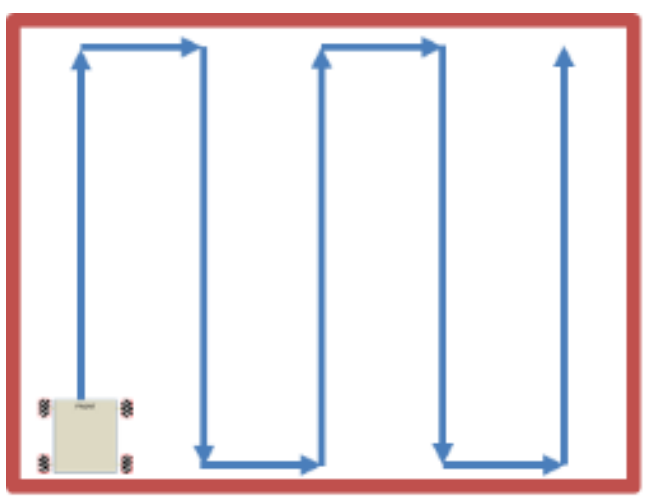

(a)

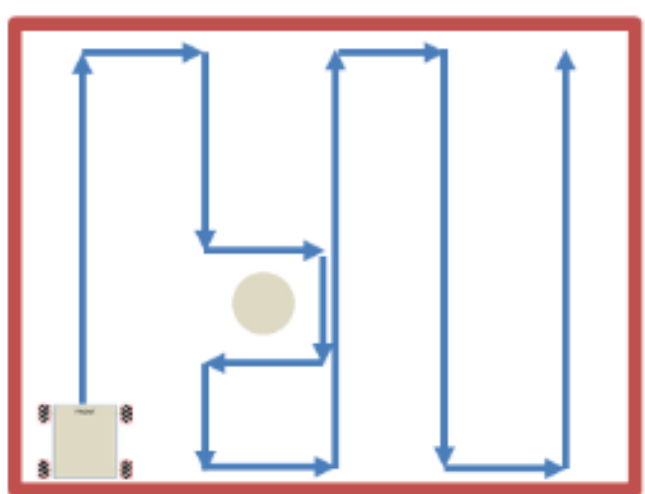

(b)

Fig. 9. The zig-zag covering strategies.

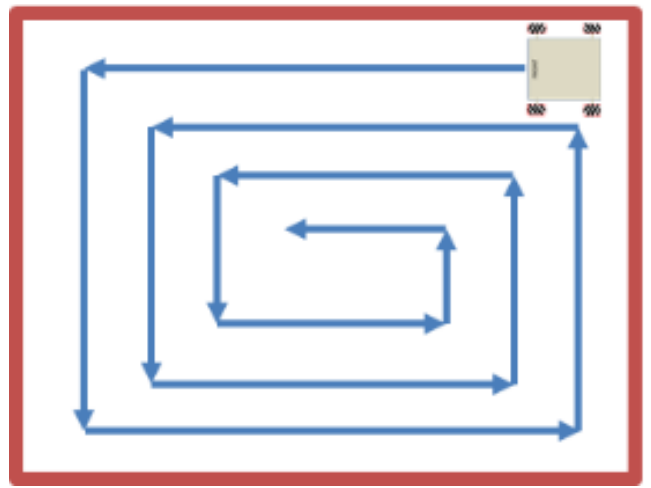

(a)

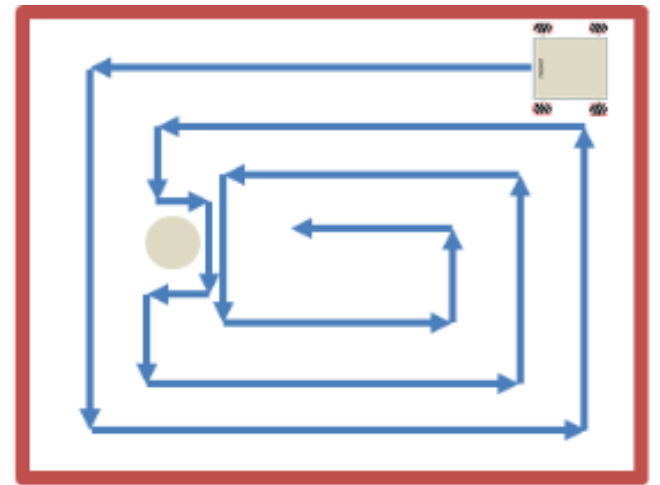

Legend:

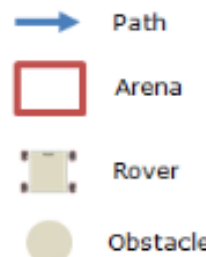

(b)

Fig. 10. The inward spiral covering strategies.

\section{Results and Discussions}

In this study, the ROS and SLAM are implemented for the vacuum cleaning robot. The dimensions of the robot are set as [ [-0.20, -0.20], $[-0.20,0.20], \quad[0.20,0.20],[0.20,-0.20]]$. The dimensions of the room are $4 \times 5 \mathrm{~m}^{2}$. The localization method is applied based on Monte Carlo algorithm. This is the algorithm that has many advantages over previous algorithms due to a significant reduction in computational burden. The approximated position of the robot in the environment is shown in Fig. 11. The robot localization is illustrated in Fig. 12. After the robot executes in the working environment, the data collected by its sensors matches the mapped data.

In the experiments, Fig.13, 14, 15 presents the results of the robot travels according to the square trajectory $(2 \mathrm{mx} 2 \mathrm{~m})$, zig-zag trajectory, and spiral trajectory, respectively. The total errors of position of different trajectories are summaried in Table 1. 
JST: Smart Systems and Devices

Volume 31, Issue 1, May 2021, 059-067

Table 1. Error position of different trajectories.

\begin{tabular}{|c|c|c|}
\hline Trajectory & Total Path & Error \\
\hline Square & $8(\mathrm{~m})$ & $\begin{array}{l}\Delta X=0.092(\mathrm{~m}) \\
\Delta Y=0.135(\mathrm{~m})\end{array}$ \\
\hline Zig-zag & $10(\mathrm{~m})$ & $\begin{array}{l}\Delta X=0.094(\mathrm{~m}) \\
\Delta Y=0.098(\mathrm{~m})\end{array}$ \\
\hline Spiral & $11(\mathrm{~m})$ & $\begin{array}{l}\Delta X=0.120(\mathrm{~m}) \\
\Delta Y=0.121(\mathrm{~m})\end{array}$ \\
\hline
\end{tabular}

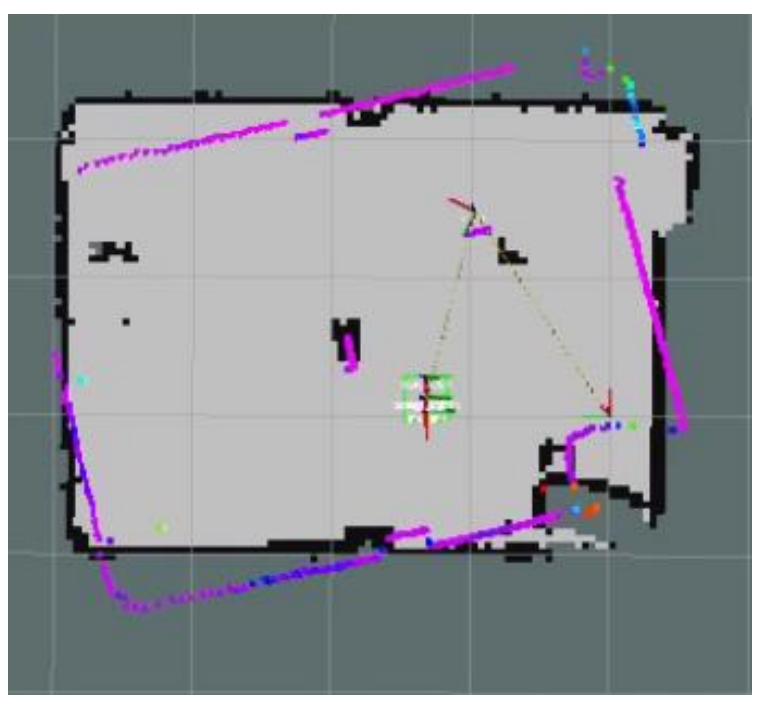

Fig. 11. Approximated position of the robot in the environment.

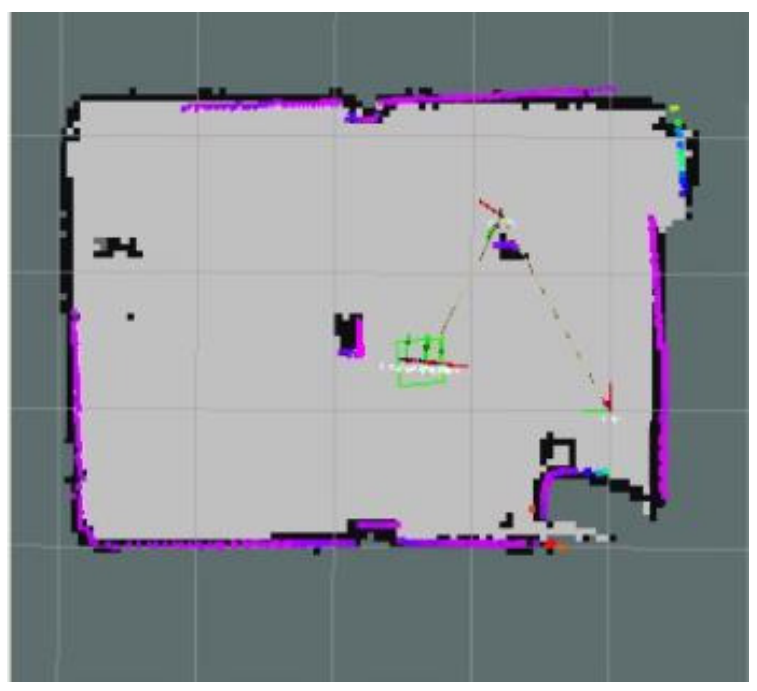

Fig. 12. Robot localization using MCL.

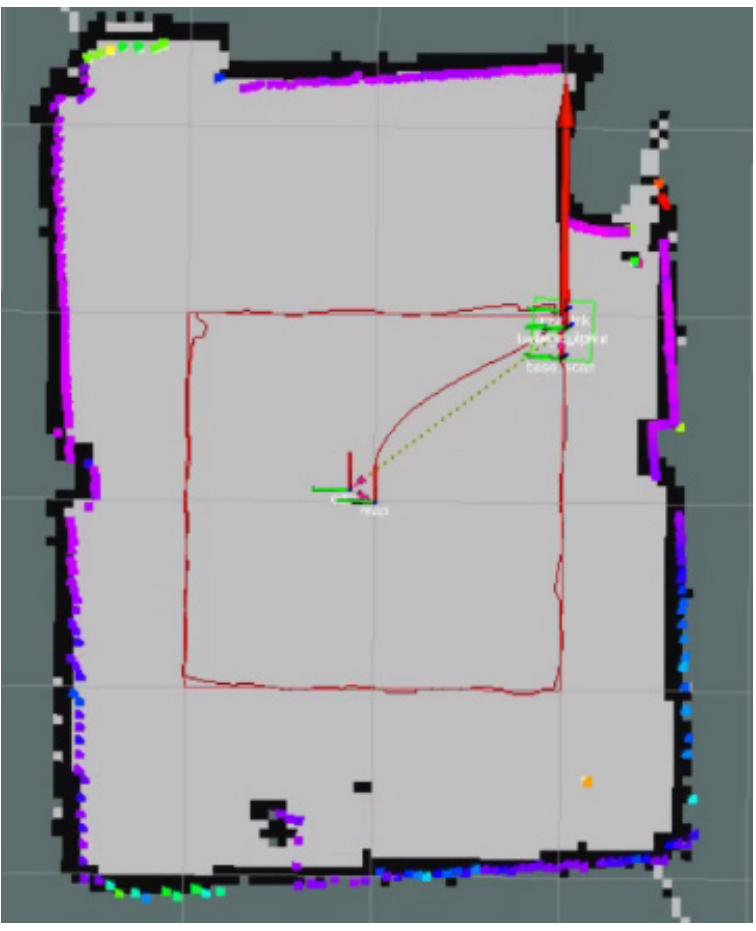

(a)

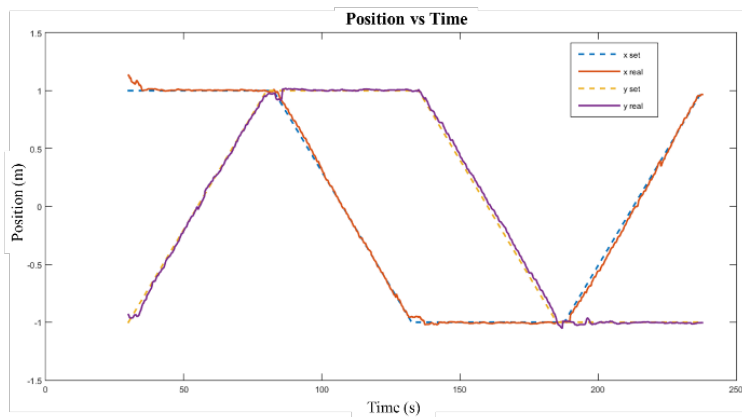

(b)

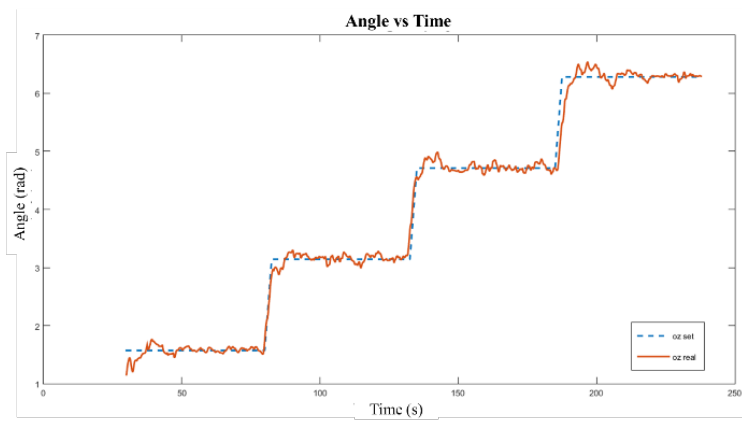

(c)

Fig. 13. (a) Square trajectory, (b) The real position of the robot in comparison with the set position, and (c) the real angle of rotation in comparison with the set angle. 


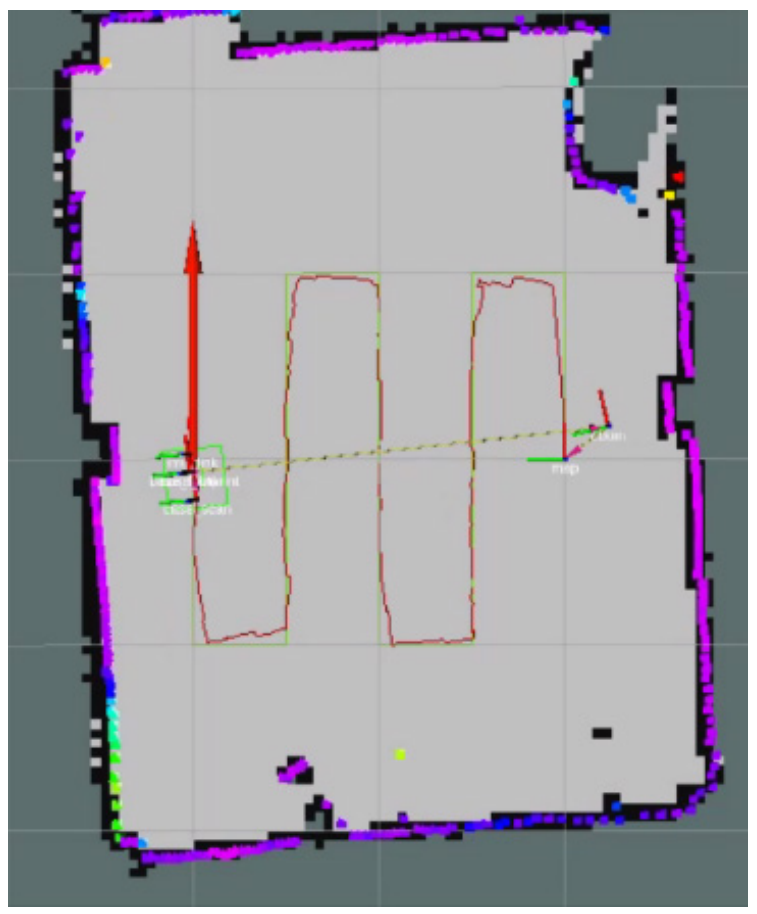

(a)

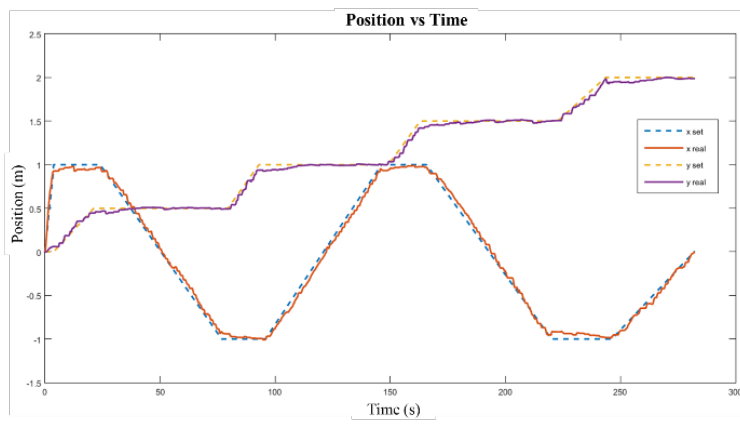

(b)

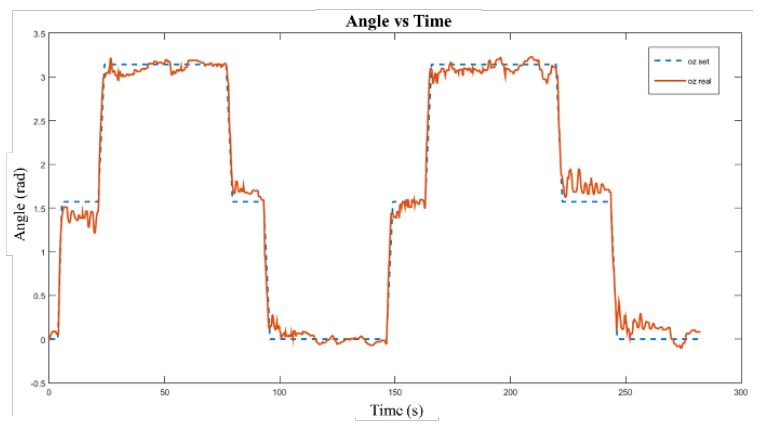

(c)

Fig. 14. (a) Zig-zac trajectory, (b) The real position of the robot in comparison with the set position, and (c) the real angle of rotation in comparison with the set angle.

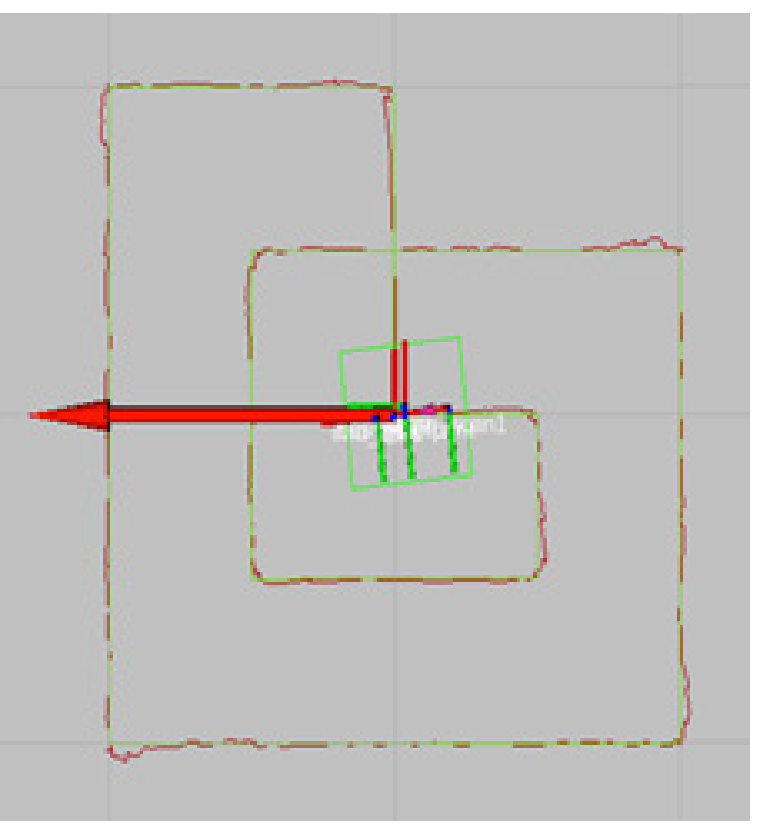

(a)

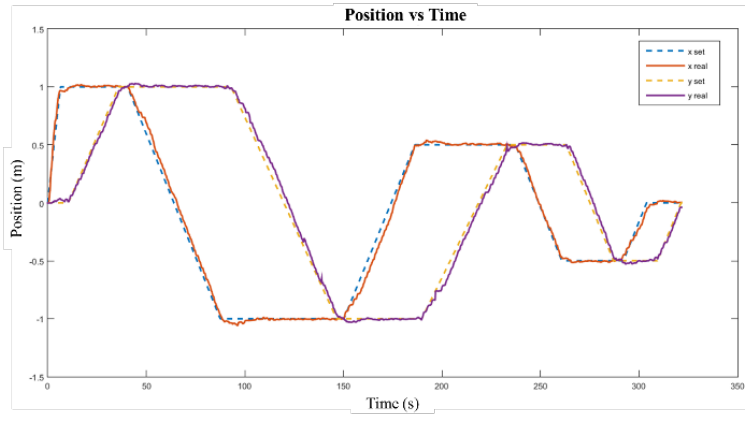

(b)

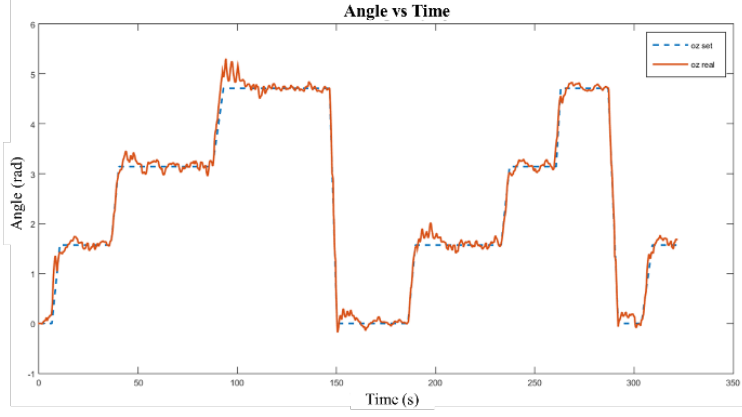

(c)

Fig. 15. (a) Spiral trajectory, (b) The real position of the robot in comparison with the set position, and (c) the real angle of rotation in comparison with the set angle.

Fig. 16 presents the mapping results in a real working environment. Fig. 17 and 18 show the covering results by the combination of the algorithms described in the subsection 2.5. In the experiments, the proposed approach always generates a suitable trajectory depending on the start position in the working environment. The covering percentage is $86 \%$. 
JST: Smart Systems and Devices

Volume 31, Issue 1, May 2021, 059-067

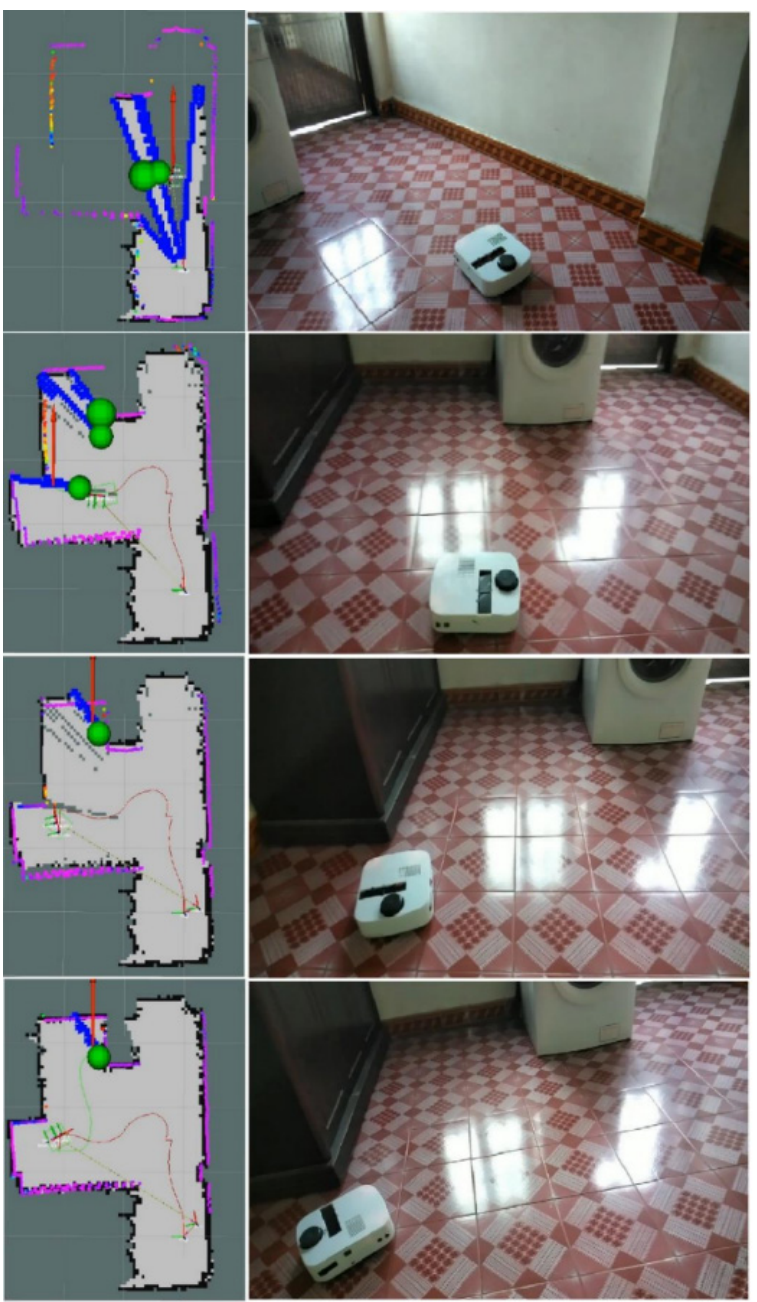

Fig. 16. Robot mapping results.

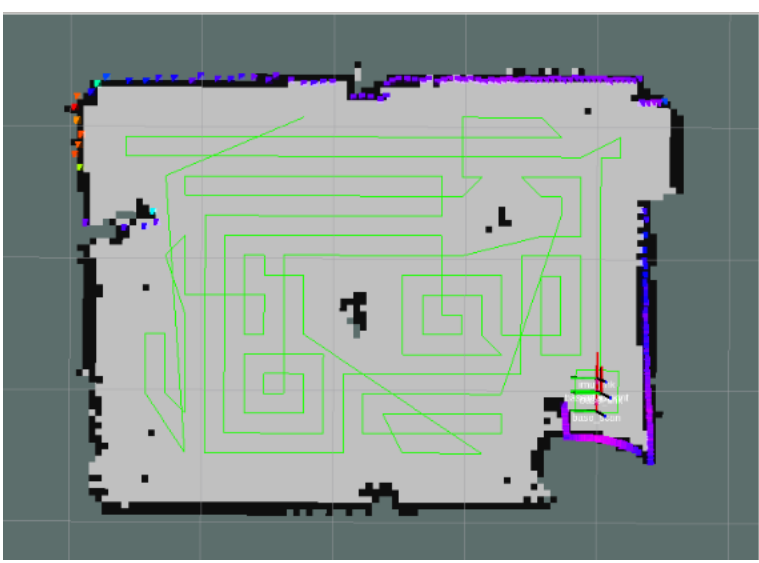

Fig. 17. Trajectory covered in real environment.

\section{Conclusion}

In this paper, we proposed an autonomous approach for a vacuum cleaner robot. The main achievements for this work are: $(i)$ the mechanical design, identification system methodology and controller development; (ii) the proposed localization method based on MCL and mapping in the ROS; (iii) the proposed covering strategy to maximum the covered floor area. The performed simulation using

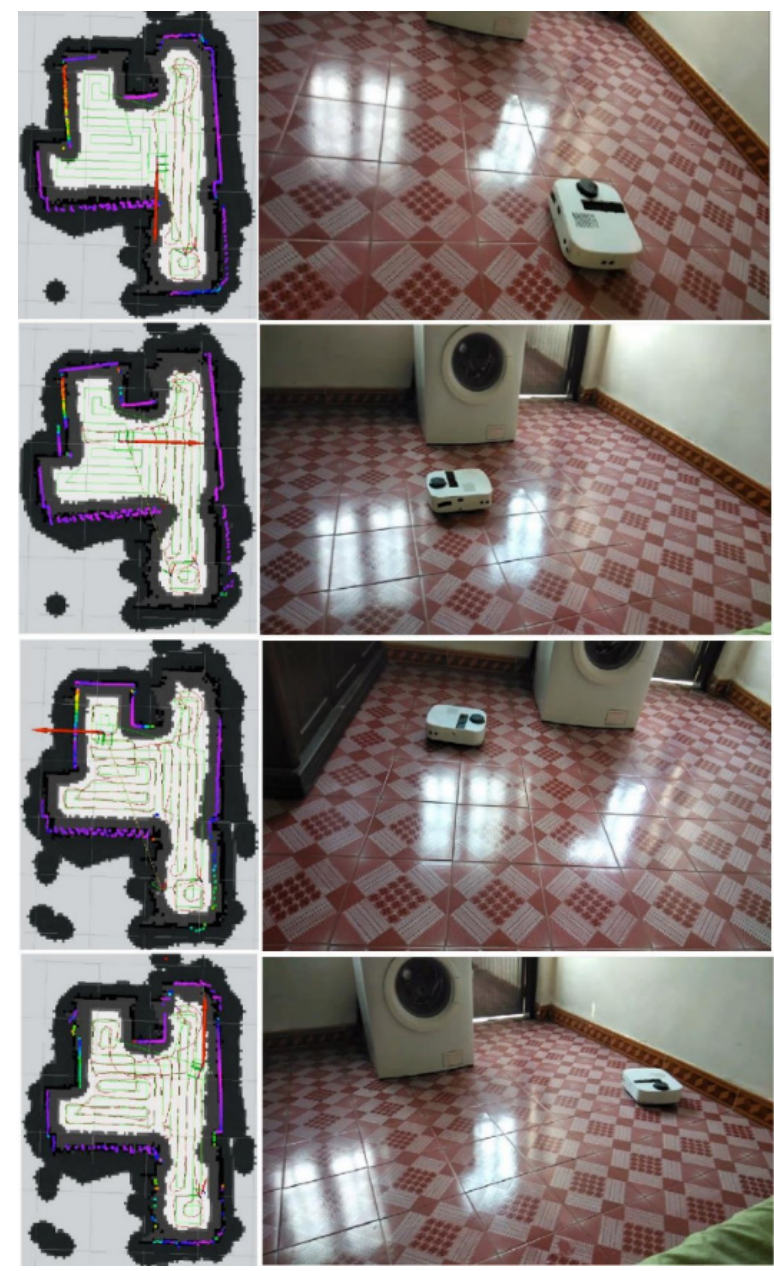

Fig. 18. Robot covering results.

the virtual environment in ROS and the real-time experiment demonstrate the feasibility of the proposed strategy. The conducted results have a covering percentage of $86 \%$.

\section{Acknowledgments}

This work is granted by the National Foundation for Science and Technology Development of Vietnam NAFOSTED, Vietnam under project number 107.012018.331.

\section{References}

[1] TT Mac, C Copot, CY Lin, HH Hai, CM Ionescu, Towards The Development of a Smart Drone Police: Illustration in Traffic Speed Monitoring, Journal of Physics: Conference Series, 2020, 12-29

[2] TT Mac, C Copot, C. M. Ionescu, Detection and Estimation of Moving obstacles for a UAV, IFACPapersOnLine, Volume 52, Issue 1, 2019, Pages 22-27. https://doi.org/10.1016/j.ifacol.2019.06.032

[3] Nicholls, Y. Strengers, Robotic vacuum cleaners save energy? Raising cleanliness conventions and energy demand in Australian households with smart home technologies, Energy Research \& Social Science, Vol. 50 (2019) 73-81.

https://doi.org/10.1016/j.erss.2018.11.019 
[4] T.B. Asafa, T.M. Afonja, E.A. Olaniyan, H.O. Alade, Development of a vacuum cleaner robot, Alexandria Engineering Journal, Vol. 57(4) (2018) 2911-2920. https://doi.org/10.1016/j.aej.2018.07.005

[5] Estela D. Vicente, Ana M. Vicente, Margarita Evtyugina, Ana I. Calvo, Fernanda Oduber, Carlos Blanco Alegre, Amaya Castro, Roberto Fraile, Teresa Nunes, Franco Lucarelli, Giulia Calzolai, Silvia Nava, Célia A. Alves, Impact of vacuum cleaning on indoor air quality, Building and Environment, Vol.180 (2020) 107059

https://doi.org/10.1016/j.buildenv.2020.107059

[6] Mohamed Amine Yakoubi, Mohamed Tayeb Laskri, The path planning of cleaner robot for coverage region using Genetic Algorithms, Journal of Innovation in Digital Ecosystems, Vol. 39(1) (2016), 37-43. https://doi.org/10.1016/j.jides.2016.05.004

[7] M. A. Yakoubi, M. T. Laskri, The path planning of cleaner robot for coverage region using Genetic Algorithms, Journal of Innovation in Digital Ecosystems, Vol.3(1) (2016) 37-43. https://doi.org/10.1016/j.jides.2016.05.004

[8] V. Prabakaran, M. Elara, T. Pathmakumar, S. Nansai, Floor cleaning robot with reconfigurable mechanism, Automation in Construction, Vol. 91 (2018) 155-165. https://doi.org/10.1016/j.autcon.2018.03.015
[9] J.Kim, A.K.Mishra, R.Limosani, M.Scafuro, N. Cauli, J. Victor, B. Mazzolai, F. Cavallo, Control strategies for cleaning robots in domestic applications: A comprehensive review, International Journal of Advanced Robotic Systems. July 2019. https://doi.org/10.1177/1729881419857432

[10] K. Zheng, G. Chen, G. Cui, Y. Chen, F. Wu, X. Chen, Performance metrics for coverage of cleaning robots with mocap system Intelligent Robotics and Applications, Springer International Publishing (2017), pp. 267-274, 10.1007/978-3-319-65298-6_25 https://doi.org/10.1007/978-3-319-65298-6_25

[11] R.J.Ong and K.N. F Ku Azir, Low Cost Autonomous Robot Cleaner using Mapping Algorithm based on Internet of Things (IoT), 2020 IOP Conf. Ser.: Mater. Sci. Eng. 767012071 https://doi.org/10.1088/1757-899X/767/1/012071

[12] F. Dellaert, D. Fox, W. Burgard and S. Thrun, Monte Carlo localization for mobile robots, Proceedings 999 IEEE International Conference on Robotics and Automation (Cat. No.99CH36288C), Detroit, MI, USA, (1999), vol.2, pp. 1322-1328. 